\title{
SLHPBA guidelines on the management of acute pancreatitis - a consensus document
}

\author{
Sivasuriya Sivaganesh ${ }^{1}$, Ajith Siriwardena ${ }^{2}$, Aloka Pathirana ${ }^{3}$ \\ ${ }^{1}$ University Surgical Unit, National Hospital of Sri Lanka, Colombo \\ ${ }^{2}$ Manchester Royal Infirmary (NHS), UK \\ ${ }^{3}$ University Surgical Unit, Colombo South Teaching Hospital, Kalubowila
}

Acute pancreatitis continues to be one of the leading causes of hospital admission for 'acute abdomen' globally including Sri Lanka. The presentation and outcome spans a wide spectrum from those who present with a mild episode that requires a few days of hospitalisation to those who develop severe disease with multi-organ dysfunction, associated local infective complications with attendant morbidity and mortality.

Similar to elsewhere in the world, in Sri Lanka, acute pancreatitis is managed by specialists that include general surgeons, gastrointestinal surgeons, general physicians and gastrointestinal physicians. In addition, radiologists, microbiologists and critical care specialists play a key role in the overall care of these patients. The concept of centralised care in pancreatic disorders is yet to be established resulting in its overall level of care being somewhat heterogeneous.

Data regarding the incidence of acute pancreatitis and its outcomes in Sri Lanka is sparse except for a handful of small unpublished studies of limited patient numbers. Reports of adverse outcomes secondary to inappropriate practice, failure to refer to specialist centres when appropriate and the lack of infrastructure and facilities have been noted.

It is in this context that the Sri Lanka Hepato-PancreatoBiliary Association (SLHPBA) sought to address the need to establish consensus guidelines to manage this important condition. In generating these guidelines, their suitability and applicability in the national health system was an important factor, since more than $80 \%$ of inpatient care is currently provided in the state health sector. The availability of resources at different levels of hospitals in terms of expertise, ancillary facilities including facilities for CT imaging, interventional radiological support and intensive care were considered when making recommendations.

These guidelines also include globally established terminology in relation to local complications of acute pancreatitis enabling all care givers to communicate via a universal language. Furthermore, this will facilitate the development of national databases and auditing of results with a view to improving quality of care and outcomes.

The scope of these guidelines is to provide evidence based recommendations for the medical and surgical management of patients with acute pancreatitis both in the acute and later phase of the disease.

The primary guideline working group consisted of members of the SLHPBA and the IHPBA (International HepatoPancreato-Biliary Association). Individuals from relevant specialities and professional organisations including the College of Surgeons of Sri Lanka, Sri Lanka Society of Gastroenterology, Sri Lanka College of Radiologist and Sri Lanka College of Microbiologists were involved in the final drafting of the guidelines.

Target users of the guidelines are all clinicians involved in the care of patients with acute pancreatitis.

The evidence and recommendations for these guidelines were derived from the following three documents:

1. IAP/APA Evidence-Based Guidelines for the Management of Acute Pancreatitis

2. The New Revised Classification of Acute Pancreatitis $2012^{2}$

3. American College of Gastroenterology Guideline: Management of Acute Pancreatitis ${ }^{3}$

The strength of recommendations and the levels of evidence for the Sri Lankan guidelines will mirror those of the above guidelines that have utilised the Grading of Recommendations Assessment, Development and Evaluation (GRADE) system. Clinicians who wish to read further with regard to the level of evidence forming the basis of the guidelines and recommendations are directed to the quoted three references.

Correspondence: Sivasuriya Sivaganesh

E-mail: sivaganesh@srg.cmb.ac.lk

Received: 15-12-2017 Accepted: 15-12-2017

(iD) http://orcid.org/0000-0002-6874-6904

DOI: http://doi.org/10.4038/sljs.v35i4.8436

The Sri Lanka Journal of Surgery 2017; 35(4): 29-33 


\section{SRI LANKA HEPATO-PANCREATO-BILIARY ASSOCIATION NATIONAL GUIDELINES 2017 MANAGEMENT OF ACUTE PANCREATITIS}

\section{A) Diagnosis}

Fulfilment of 2 out of 3 of the following criteria (usually $1 \& 2)$ :

1. Central upper abdominal pain usually of acute onset often radiating to the back

2. Serum amylase or lipase activity $>3$ times the upper limit of normal

3. Characteristic features on cross-sectional (CT / MRI) abdominal imaging

Note:

I. Serum amylase and lipase are primarily of diagnostic value and not indicators of disease severity. Hence, repeat assays are not indicated once a diagnosis is established.

ii. Optimal timing for initial CT assessment, if required, is at least 72 - 96 hours after onset of symptoms.

However, indications for early abdominal CECT in AP include:

1. Uncertainty of diagnosis in terms of clinical presentation e.g. suspected visceral perforation

2. Equivocal biochemistry in the presence of typical clinical picture

3. Clinical deterioration despite optimal care within $48-72$ hours of admission

\section{B) Assessment of severity}

It is recommended that severity may be assessed using one of the methods a) or b) given below:

\section{a) Organ failure based on Modified Marshall Score}

\section{Mild AP}

- No organ failure

- Lack of local or systemic complications

\section{Moderate AP}

- Organ failure that resolves within 48 hours (transient organ failure) and/or

- Local or systemic complications (sterile or infected) without persistent organ failure

\section{Severe AP}

- Persistent single or multiple organ failure ( $>48$ hours)

\section{Definition of organ failure}

Table 1. Modified Marshall Score

A score $\geq \mathbf{2}$ in any system defines the presence of organ failure

\begin{tabular}{|l|c|c|c|c|c|}
\hline & $\mathbf{0}$ & $\mathbf{1}$ & $\mathbf{2}$ & $\mathbf{3}$ & $\mathbf{4}$ \\
\hline $\begin{array}{l}\text { Respiratory } \\
\mathrm{PaO}_{2} / \mathrm{FiO}_{2}\end{array}$ & $>400$ & $301-400$ & $201-300$ & $101-200$ & $\leq 100$ \\
\hline $\begin{array}{l}\text { Renal } \\
\text { (serum } \\
\text { creatinine) } \\
\mu \mathrm{mol} / \mathrm{L}\end{array}$ & $\leq 134$ & $134-169$ & $170-310$ & $311-439$ & $>439$ \\
\hline $\mathrm{mg} / \mathrm{dl}$ & $<1.4$ & $1.4-1.8$ & $1.9-3.6$ & $3.7-4.9$ & $\geq 5$ \\
\hline $\begin{array}{l}\mathrm{CVS} \\
\mathrm{SBP} \mathrm{mmHg}\end{array}$ & $>90$ & $\begin{array}{c}<90 \text { fluid } \\
\text { responsive }\end{array}$ & $\begin{array}{c}<90 \text { non } \\
\text { responsive } \\
\text { to fluid }\end{array}$ & $\begin{array}{l}\mathrm{pH}<7.3 \\
\mathrm{pH}<7.2\end{array}$ \\
\hline
\end{tabular}

- Scoring patients with pre-existent CKD depends on the extent of deterioration over baseline renal function. Calculations for a baseline serum creatinine $\geq 134$ $\mathrm{mmol} / \mathrm{L}$ or $1.4 \mathrm{mg} / \mathrm{dL}$ are not available.

- $\mathrm{SBP}$ - off inotropic support

b) Severity based on systemic inflammatory response syndrome (SIRS)

SIRS is defined by the presence of two (2) or more of the following four (4) criteria:

(1) Temperature $<36^{\circ} \mathrm{C}\left(96.8^{\circ} \mathrm{F}\right)$ or $>38^{\circ} \mathrm{C}\left(100.4^{\circ} \mathrm{F}\right)$

(2) Heart rate $\quad>90 / \mathrm{min}$

(3) Respiratory rate $>20 / \mathrm{min}$

(4)White blood cells $<4000 / \mathrm{mm}^{3},>12000 / \mathrm{mm}^{3}$ or $10 \%$ band forms

Severe acute pancreatitis in indicated by SIRS at admission and persistent SIRS at $48 \mathrm{~h}$

Note:

Other indicators of severe AP at admission or $72 \mathrm{~h}$

- $\mathrm{CRP} \geq \mathbf{1 5 0} \mathrm{mg} / \mathrm{dL}$

\section{Local complications (see annexure for definitions)}

1. Acute peripancreatic fluid collection (APFC)

2. Pancreatic pseudocyst

3. Acute necrotic collection (ANC)

4. Walled off necrosis (WON)

5. Gastric outlet obstruction

6. Splenic/portal vein thrombosis

7. Retroperitoneal haemorrhage

8. Pancreatic ascites

9. Pancreatico-pleural fistula 


\section{C) Initial assesment and risk stratification}

1. Haemodynamic status should be assessed immediately upon presentation and resuscitative measures begun as needed

2. Baseline investigations on admission:

FBC, CRP, BU, creatinine, electrolytes, AST / ALT, bilirubin, ALP

ABG (in haemodynamically abnormal / oliguric / tachypnoeic patients)

3.Ultrasonography should be performed in all patients within 24 hours to evaluate the biliary tract for:

- Gallstones

- Common bile duct(CBD) calculi

- Biliary tract dilatation

4. Risk stratification is required to

- Predict outcome of AP

- Determine those who require HDU / ICU care

5. A 3-dimensional approach to risk stratification is recommended:

- Host risk factors (e.g. age, co-morbidity, body mass index)

- Disease severity (mild / moderate / severe)

- Response to initial therapy (clinical \& biochemical)

\section{Admission to an HDU or ICU is recommended in}

- Evolving or established organ failure (moderate - severe AP)

- Elderly, multiple co-morbidities

\section{D) Management}

\section{Initial fluid therapy}

a. Aggressive hydration, defined as $\mathbf{5 - 1 0 ~} \mathrm{ml} / \mathbf{k g} / \mathbf{h}$ of isotonic crystalloid solution should be provided to all patients, unless cardiovascular and/or renal co-morbidities exist. Early aggressive intravenous hydration is most beneficial the first $12-24 \mathrm{~h}$, and may have little benefit beyond

b. In patients with severe volume depletion, manifest as hypotension and tachycardia, more rapid repletion (bolus) may be needed

c. Recommended fluid: Hartmann (Ringer's lactate) solution

d. Fluid therapy should be goal directed and reassessed at frequent intervals within $6 \mathrm{~h}$ of admission and for the next $24-48 \mathrm{~h}$.

e. The goals of initial fluid therapy:

- Heartrate <120 bpm
- MAP 65 - $85 \mathrm{mmHg}$

- Urine output $0.5-1.0 \mathrm{ml} / \mathrm{kg} / \mathrm{hr}$

- Haematocrit 35-40\%

- Blood urea reduction

Note: trends in parameters are important

\section{Pain relief}

Multimodal analgesic regimens are recommended

- Paracetamol-suppositories

- Opioids-Morphine IV/SC

- NSAIDs (caution in renal impairment)

\section{Antibiotics}

a. Routine use of prophylactic antibiotics in patients with mild, moderate or severe AP is not recommended

b. The use of antibiotics in patients with sterile necrosis to prevent the development of infected necrosis is not recommended

c. Routine administration of antifungal agents is not recommended

d. In patients with suspected infected necrosis, antibiotics known to penetrate pancreatic necrosis, such as carbapenems, quinolones, and metronidazole, may be useful in delaying or sometimes totally avoiding intervention, thus decreasing morbidity and mortality

e. Antibiotics should be given for an extra-pancreatic infection, such as cholangitis, catheter-acquired infections, bacteraemia, urinary tract infections, pneumonia

\section{Nutrition}

a. In mild AP, oral feeds can be started immediately if there is no significant pain, nausea and vomiting

b. In mild AP, initiation of feeding with a low-fat solid diet appears as safe as a clear liquid diet

c. In severe AP, nasogastric / jejunal enteral nutrition is recommended to prevent infectious complications

d. Polymeric formulations are as effective as elemental for-mulations for enteral nutrition in AP

e. Nasogastric and nasojejunal enteral feeding appear comparable in efficacy and safety

f. Parenteral nutrition is instituted in AP only if enteral (NG/NJ) feeding is not tolerated and fails to meet nutritional requirement

\section{ERCP (endoscopic retrograde cholangio pancreatico- graphy)}

a. Patients with AP and

- concurrent acute cholangitis or

- biliary tract obstruction should undergo urgent ERCP within $24 \mathrm{~h}$ of admission 
b. Pancreatic duct stents and / or post-procedure rectal NSAID suppositories should be utilized to prevent severe post-ERCP pancreatitis in high-risk patients

c. In unstable and if ERCP is not safely feasible, placement of a percutaneous transhepatic (PTC) biliary drainage tube should be considered

d. ERCP is not needed in most patients with gallstone pancreatitis who lack laboratory or imaging evidence of ongoing biliary obstruction

e. If choledocholithiasis is strongly suspected in the absence of cholangitis and/or jaundice, MRCP or endoscopic ultrasound (EUS) is recommended

\section{Cholecystectomy}

a. In patients with mild AP with gallstones, a laparoscopic cholecystectomy should be performed during the index admission before discharge to prevent a recurrence of AP

b. If a cholecystectomy cannot be done during the index admission it should be done within 2 weeks of discharge

c. In a patient with severe or necrotizing gallstone AP, in order to prevent infection, cholecystectomy is to be deferred until active inflammation (clinical \& biochemical) subsides and fluid collections resolve or stabilize

\section{Management of local complications}

a.The presence of asymptomatic pseudocysts and pancreatic and/or extra-pancreatic necrosis do NOT warrant intervention, regardless of size, location, and/or extension

b. Indications for intervention (radiological / endoscopic / surgical) in sterile necrotizing AP are:

- Ongoing gastric outlet, intestinal, or biliary obstruction due to mass effect of WON (i.e. arbitrarily $>4$ - 8weeks after onset of AP)

- Persistent symptoms (e.g. pain, 'persistent unwellness') in patients with WON without signs of infection (i.e. arbitrarily $>8$ weeks after onset of AP)

- Disconnected duct syndrome (i.e. full transection of the pancreatic duct in the presence of pancreatic necrosis) with persisting symptomatic (e.g. pain, obstruction) collection(s) with necrosis without signs of infections (i.e. arbitrarily $>8$ weeks after onset of AP)

- Ongoing organ failure for several weeks after the onset of AP, preferably after WON

c. Other indications for emergency surgery without concurrent necrosectomy

- Abdominal compartment syndrome

- Ongoing acute bleeding

- Bowel ischaemia

\section{Diagnosis \& treatment of infected necrosis}

a. Infected necrosis should be considered in patients with pancreatic or peripancreatic necrosis who deteriorate or fail to improve after 7 - 10 days (2nd week onwards) of hospitalization.

b. It is diagnosed by:

- Clinical features of ongoing sepsis

- CECT - extraluminal gas in pancreatic or peripancreatic necrotic tissue

- Percutaneous FNA for microbiological analysis - not recommended

c. In stable patients with infected necrosis, surgical, radiological, and/or endoscopic drainage should be delayed preferably for more than 4 weeks to allow liquefaction of the contents and the development of a fibrous wall around the necrosis (walled-off necrosis)

d. In symptomatic patients with infected necrosis, minimal access methods (percutaneous / endoscopic transluminal) are preferred to open necrosectomy

e. Patients with

- extensive necrotizing acute pancreatitis

- who show no clinical signs of improvement following appropriate initial management

- who experience other complications

- should be managed in institutions that have on-site or access to therapeutic endoscopy, interventional radiology, surgeons and intensivists with expertise in dealing with severe acute pancreatitis.

\section{Determination of aetiology}

a. In the absence of gallstones and/or history of significant history of alcohol use, a detailed history should be obtained to look for aetiological factors

b. Serum triglyceride should be obtained and considered the aetiology if $>1,000 \mathrm{mg} / \mathrm{dl}$

c. Serum ionised calcium if above normal should be investigated for primary hyperparathyroidism

d. In patients $>\mathbf{4 0}$ years, a pancreatic tumour should be considered and a CECT performed

e. Patients with idiopathic pancreatitis should be referred to centres of expertise

f. Genetic testing may be considered in young patients $(<30$ years old) if no cause is evident and a family history of pancreatic disease is present 


\section{Definitions}

\section{Types of AP}

\section{Interstitial oedematous pancreatitis}

Inflammation of pancreatic parenchyma and peripancreatic tissue, but without obvious tissue necrosis.

- Enhancement of the pancreatic parenchyma by contrast agent

- No evidence of peripancreatic necrosis (see below)

\section{Necrotizing pancreatitis}

- Inflammation with pancreatic parenchymal necrosis and/or peripancreatic necrosis.

- Areas of pancreatic parenchymal lacking by intravenous contrast agent and/or

- Findings of peripancreatic necrosis (see below-ANC and WON)

\section{Local complications}

\section{Acute peripancreatic fluid collection (APFC)}

Peripancreatic fluid with interstitial oedematous pancreatitis and no peripancreatic necrosis. This term applies to peripancreatic fluid seen within the first 4 weeks after the onset of interstitial oedematous pancreatitis.

- Homogeneous collection with fluid density adjacent to pancreas confined by normal

- peripancreatic fascial planes

- No recognizable wall encapsulating the collection

- Occurs only in interstitial oedematous pancreatitis

\section{Pancreatic pseudocyst}

Encapsulated fluid collection with minimal or no necrosis with a well-defined inflammatory wall usually outside the pancreas. This entity occurs more than 4 weeks after the onset of interstitial oedematous pancreatitis.

- Round or oval well circumscribed, homogeneous fluid collection

- No non-liquid component

- Well-defined wall

- Occurs after interstitial oedematous pancreatitis

\section{Acute necrotic collection (ANC)}

A collection of both fluid and necrosis associated with necrotizing pancreatitis involving the pancreatic parenchyma and/or the peripancreatic tissues

- Heterogeneous, non-liquid density of varying degrees

- No definable encapsulating wall

- Location: intrapancreatic and/or extrapancreatic

- Occurs in setting of acute necrotizing pancreatitis

\section{Walled-off necrosis (WON)}

A mature, encapsulated collection of pancreatic and/or peripancreatic necrosis with a well-defined inflammatory wall occurring more than 4 weeks after the onset of necrotizing pancreatitis.

- Heterogeneous liquid and non-liquid density with varying degrees of loculations

- Well-defined encapsulating wall

- Location: intrapancreatic and/or extrapancreatic

- Occurs only in setting of necrotizing pancreatitis

\section{References}

1. IAP / APA evidence - based guidelines for the management of acute pancreatitis. Working Group IAP/APA Acute Pancreatitis Guidelines 2013. Pancreatology 13 (2013) e1 - e15. doi.org/10.1016/j.pan.2013.07.063

2. The New Revised Classification of Acute Pancreatitis. M G Sarr et al. 2012 Surg Clin N Am 93 (2013) 549-562 doi.org/10.1016/j.suc.2013.02.012

3. American College of Gastroenterology Guideline: Management of Acute Pancreatitis. S Tenner, J Baillie, J DeWitt, S S Vege. Am $J$ Gastroenterol July 2013; doi.org/10.1038/ajg.2013.218

\section{Acknowledgement}

The primary guideline working group consisted of members of the SLHPBA and the IHPBA (International Hepato - Pancreato - Biliary Association). Individuals from relevant specialities and professional organisations including the College of Surgeons of Sri Lanka, Sri Lanka Society of Gastroenterology, Sri Lanka College of Radiologist and Sri Lanka College of Microbiologists were involved in the final drafting of the guidelines. 